\title{
Strategies of Receivables Management in Multi-entity Organizations
}

\author{
Grzegorz Zimon \\ Department of Finance, Banking and Accounting \\ Rzeszow University of Technology \\ Rzeszow, Poland \\ gzimon@prz.edu.pl
}

\begin{abstract}
Receivables management is the most important area of financial management. Their level and a management policy have a decisive impact on the financial safety of enterprises. Receivables are the basic component of current assets. In general, short-term receivables (current receivables), i.e. receivables from customers are the largest share in the structure of receivables. Managers are still looking for some tools, methods of managing them, which will enable them to manage such a policy that will allow their quick impact without the risk of losing a contractor. Consumers operating in the functioning market gain large opportunities to manage receivables from customers in group purchasing organizations, capital groups and clusters. Joint operation allows using certain tools and methods that improve the receivables management process.

The aim of the article is to present the strategy of managing receivables in group purchasing organizations. They were divided into branch and multi-branch ones. Purchasing groups are most often associated with the purchase process, the effect of scale. However, the joint action has a strong impact on building a receivables management strategy. The analysis was carried out on a group of 70 Polish small trade enterprises. The research was carried out on the basis of financial statements for the years 2014-2016. Selected financial ratios and statistical methods were used for the research.
\end{abstract}

Keywords-Receivables; Group purchasing organizations (GPOs); Strategies

\section{INTRODUCTION}

Receivables management is a process that begins much before an invoice, i.e. a deferred payment account is issued. In order to manage receivables from recipients effectively, some appropriate rules of granting trade credit should be introduced in advance. The contractors should be divided into appropriate risk groups. It is necessary to discuss lending limits and the length of trade credits for each risk group. The created policy of managing receivables simplifies the process of debt collection and minimizes the risk of bad debts. The sales department should act strictly according to the rules that are set out in the receivables management policy at the time of the sales transaction. Otherwise, such a transaction is subject to high risk. Decisions on granting trade credit are of great importance for sales volumes and maintaining financial liquidity [1]. Trade credit is a necessity today, every company must offer it to its recipients. Receivables are a form of crediting customers, which can be an excellent "weapon" to fight against competition [2]. International competition and the high number of newly created companies in a given area are the main reasons for the financial problems of enterprises [3] Extending payment terms encourages buyers to make purchases. Receivables management is a process whose main assumption is to counteract the occurrence of overdue receivables. An enterprise that manages receivables efficiently is competitive in the market as it has free cash. It can pay faster to its contractors, which improves its image in the market. Such a company is reliable, it can offer contractors more favorable conditions for granting trade credits. In the case of receivables three basic strategies: conservative, moderate and aggressive can be distinguished [4]. The conservative strategy is based on the creation of such mechanisms that will completely eliminate the risk of customer insolvency. In the case of this strategy contractors who do not pay their obligations are quickly abandoned. This strategy is based on continuous monitoring of receivables and an aggressive debt collection. Such rules may discourage contractors from cooperation, which will have a negative impact on the level of revenues in the future. When customers give up, the revenues will decrease. The aggressive strategy is based on excessive trust towards recipients. Sales with deferred, often long-term payments are carried out for trusted and new contractors. If the recipients are honest and have no financial problems, then it results in high revenues and profits. However, if bad debts appear and continue growing, then in the long-term the aggressive strategy will lead to the bankruptcy of the enterprise. The last of the classic strategies is the moderate strategy which is an indirect solution in the case of receivables management. Moderate strategies are worth separating into moderate-conservative and moderate aggressive. In the case of the moderate-conservative strategy, the basic rules of conduct should be those that apply to the conservative strategy with one exception. Managers should introduce cautious debt collection not to discourage contractors. They should offer some discounts for early payment and limit aggressive methods of collection of receivables and take some risk of the possibility of occurrence of overdue receivables. These activities should be selected so that they do not lead to the loss of contractors. The moderate-aggressive strategy based on the aggressive one should eliminate the sales of cash to new contractors. Alternatively, a 2-3 day payment period should be introduced. Conducting sales based on granting a long trade credit will certainly lead to payment bottlenecks. 


\section{GROUP PURCHASING ORGANIZATIONS VS. RECEIVABLES}

The easiest way to describe a group purchasing organization is as a group of companies from the same or different branch that combine to make joint purchases .Another definition defines the purchasing group as a group of cooperating enterprises. They are managed by a specially created central unit whose purpose is to carry out tasks commissioned by enterprises forming a purchasing group [5]. In the case of the functioning of purchasing groups, trust and cooperation are very important. Consolidation of members of a given group is the basis for negotiations with producers [6]. The purchasing group can be described as powerful buyers. They meet important criteria characteristic of powerful buyers, they buy large quantities [7]. And the fact that companies make large purchases and use the economies of scale makes that the management of receivables in purchasing groups largely depends on the purchasing power of the group. In their research, the authors, as the most important benefits offered by functioning in purchasing groups, mention a reduction in the prices of purchased goods [8], reduction of administrative costs $[9,10]$ and production costs [11]. Benefits also arise when managing receivables from customers, which positively affects financial liquidity.

In the case of multi-entity organizations, which include purchasing groups, the willingness to cooperate within the group is important. Enterprises should exchange experiences, information and knowledge. Only those companies that cooperate within the purchasing group are able to use certain tools that positively influence the receivables management process. The group of these methods and tools include:

- Group purchases, thanks to joint purchases of the company, in addition to the favorable price, they receive an attractive merchant credit. The long period of this loan gives enterprises an opportunity to extend the loan period to the recipients. This action allows maintaining your existing customers and it is also a tool to fight for new business partners.

- Benchmarking, which is the process of learning from the best and comparing with them. It is an effective tool supporting the process of managing receivables. Benchmarking is difficult to implement if we try to compare with a direct competitor due to problems with obtaining the information needed. In purchasing groups this barrier should be eliminated because companies are trying to cooperate with each other.

- Joint monitoring of receivables, information flow about unreliable customers should be the foundation for building individual receivables management strategies. The creation of such databases in branch groups means persistence and a great simplification for enterprises.

- Mutual transactions in the purchasing group are transactions between the central unit and the enterprise or between any enterprises operating in the purchasing group. These transactions are most often introduced to help a company that is in financial trouble at the moment. In a branch purchasing group companies have more opportunities to help themselves because they buy the same goods from the same suppliers.

In the case of purchasing groups it is important to divide them into multi-branch and branch ones [12]. The branch groups only include units from the same branch. It is easier to manage them, and most importantly, the number of suppliers the negotiations are conducted with is limited. This restriction strengthens the scale effect, there is no fragmentation of participants of the purchasing group on individual recipients. In turn, in the multi-branch purchasing group there are problems with the multi-discipline of participants. There are many suppliers, companies are separated. Table I shows which tool can be used for a specific purchasing group.

TABLE I. AN IMPACT OF TOOLS SUPPORTING THE PROCESS OF MANAGING RECEIVABLES FOR THE FUNCTIONING OF AN ENTERPRISE

\begin{tabular}{|l|l|l|}
\hline $\begin{array}{c}\text { Tool of receivables } \\
\text { management }\end{array}$ & \multicolumn{1}{|c|}{ Branch group } & \multicolumn{1}{|c|}{$\begin{array}{c}\text { Multi-branch } \\
\text { group }\end{array}$} \\
\hline Group purchases & $\begin{array}{l}\text { High impact and high } \\
\text { benefits }\end{array}$ & $\begin{array}{l}\text { Low benefits due to } \\
\text { the division into } \\
\text { individual suppliers }\end{array}$ \\
\hline Benchmarking & $\begin{array}{l}\text { High impact and high } \\
\text { benefits limited to } \\
\text { only one branch }\end{array}$ & $\begin{array}{l}\text { High benefits, the } \\
\text { possibility of } \\
\text { comparison with } \\
\text { units from a different } \\
\text { branch }\end{array}$ \\
\hline $\begin{array}{l}\text { Joint receivables } \\
\text { monitoring }\end{array}$ & $\begin{array}{l}\text { High impact and high } \\
\text { benefits }\end{array}$ & $\begin{array}{l}\text { Low benefits, } \\
\text { enterprises operate in } \\
\text { various branches. } \\
\text { They have } \\
\text { completely different } \\
\text { recipients. }\end{array}$ \\
\hline Mutual transactions & $\begin{array}{l}\text { Big opportunities and } \\
\text { high benefits }\end{array}$ & $\begin{array}{l}\text { There are no such } \\
\text { possibilities. Mutual } \\
\text { transactions are only } \\
\text { possible in in } \\
\text { companies in the } \\
\text { same branch. }\end{array}$ \\
\hline
\end{tabular}

The use of the above methods and tool supporting the receivables management process affects positively the receivables management policy.

\section{Methodology And SubJect Of Research}

The research covered 70 Polish small trading enterprises operating in group purchasing organizations. The enterprises belonged to the branch purchasing groups and they were wholesalers operating in the construction industry. They generate a turnover in the range from PLN 10 million (USD 2 million) to PLN 120 million.(USD 30 million). The research period covered the years 2014-2016. The analysis was concerned the evaluation of the receivables management strategy. For this purpose the selected financial ratios and statistical methods were used as basic tools.

\section{ThE RESUlts Of ThE ANALYsis}

In order to determine the strategy for managing receivables in purchasing groups, the ratio of the share of receivables in current assets and the receivables turnover rate in days were used. The detailed results for enterprises operating in branch and multi-branch groups are presented in Tables II and III. 
TABLE II. THE SHARE OF RECEIVABLES AND RECEIVABLES TURNOVER IN THE SURVEYED ENTERPRISES FROM BRANCH GROUPS IN THE PERIOD 20142016

\begin{tabular}{|c|c|c|c|c|c|c|}
\hline $\begin{array}{l}\text { Enterp } \\
\text { rise }\end{array}$ & $\begin{array}{c}2016 \\
\text { Receiv- } \\
\text { able } \\
\text { Share }\end{array}$ & $\begin{array}{c}2015 \\
\text { Receiv- } \\
\text { able } \\
\text { Share }\end{array}$ & $\begin{array}{c}2014 \\
\text { Receiv- } \\
\text { able } \\
\text { Share }\end{array}$ & $\begin{array}{c}2016 \\
\text { Receiv- } \\
\text { able } \\
\text { turnov } \\
\text { er ratio }\end{array}$ & $\begin{array}{c}2015 \\
\text { Receiv- } \\
\text { able } \\
\text { turnov } \\
\text { er ratio }\end{array}$ & $\begin{array}{c}2014 \\
\text { Receiv- } \\
\text { able } \\
\text { turnov } \\
\text { er ratio }\end{array}$ \\
\hline 1 & 0.52 & 0.50 & 0.45 & 70 & 59 & 57 \\
\hline 2 & 0.16 & 0.13 & 0.14 & 42 & 32 & 50 \\
\hline 3 & 0.56 & 0.55 & 0.56 & 72 & 73 & 75 \\
\hline 4 & 0.56 & 0.54 & 0.57 & 80 & 76 & 81 \\
\hline 5 & 0.46 & 0.47 & 0.48 & 78 & 83 & 69 \\
\hline 6 & 0.70 & 0.71 & 0.74 & 132 & 113 & 122 \\
\hline 7 & 0.50 & 0.48 & 0.50 & 79 & 76 & 83 \\
\hline 8 & 0.63 & 0.64 & 0.58 & 84 & 74 & 89 \\
\hline 9 & 0.38 & 0.50 & 0.49 & 51 & 60 & 58 \\
\hline 10 & 0.44 & 0.32 & 0.29 & 58 & 38 & 31 \\
\hline 11 & 0.39 & 0.45 & 0.42 & 80 & 77 & 77 \\
\hline 12 & 0.09 & 0.13 & 0.17 & 11 & 14 & 18 \\
\hline 13 & 0.36 & 0.48 & 0.43 & 61 & 74 & 62 \\
\hline 14 & 0.50 & 0.48 & 0.53 & 68 & 55 & 69 \\
\hline 15 & 0.30 & 0.34 & 0.35 & 70 & 77 & 77 \\
\hline 16 & 0.56 & 0.53 & 0.57 & 81 & 76 & 60 \\
\hline 17 & 0.35 & 0.47 & 0.51 & 56 & 65 & 65 \\
\hline 18 & 0.55 & 0.57 & 0.58 & 92 & 83 & 83 \\
\hline 19 & 0.55 & 0.56 & 0.53 & 90 & 99 & 91 \\
\hline 20 & 0.16 & 0.20 & 0.20 & 35 & 29 & 25 \\
\hline 21 & 062 & 0.48 & 0.59 & 90 & 79 & 88 \\
\hline 22 & 0.47 & 0.43 & 0.40 & 64 & 66 & 62 \\
\hline 23 & 051 & 0.51 & 0.50 & 73 & 57 & 52 \\
\hline 24 & 0.48 & 0.49 & 0.52 & 61 & 58 & 65 \\
\hline 25 & 0.60 & 0.66 & 0.57 & 98 & 88 & 89 \\
\hline 26 & 0.57 & 0.59 & 0.62 & 94 & 92 & 102 \\
\hline 27 & 0.46 & 0.48 & 0.42 & 97 & 90 & 77 \\
\hline 28 & 0.56 & 0.62 & 0.56 & 84 & 88 & 78 \\
\hline 29 & 0.52 & 0.50 & 0.45 & 70 & 59 & 57 \\
\hline 30 & 0.16 & 0.13 & 0.14 & 42 & 32 & 50 \\
\hline 31 & 0.56 & 0.55 & 0.56 & 72 & 73 & 75 \\
\hline 32 & 0.56 & 0.54 & 0.57 & 80 & 76 & 81 \\
\hline 33 & 0.46 & 0.47 & 0.48 & 78 & 83 & 69 \\
\hline 34 & 0.70 & 0.71 & 0.74 & 132 & 113 & 122 \\
\hline 35 & 0.50 & 0.48 & 0.50 & 79 & 76 & 83 \\
\hline
\end{tabular}

TABLE III. THE SHARE OF RECEIVABLES AND RECEIVABLES TURNOVER IN THE SURVEYED ENTERPRISES FROM MULTI-BRANCH GROUPS IN THE PERIOD 2014-2016

\begin{tabular}{|c|c|c|c|c|c|c|}
\hline $\begin{array}{c}\text { Enterp } \\
\text { rise }\end{array}$ & $\begin{array}{c}2016 \\
\text { Receiv- } \\
\text { able } \\
\text { Share }\end{array}$ & $\begin{array}{c}2015 \\
\text { Receiv- } \\
\text { able } \\
\text { Share }\end{array}$ & $\begin{array}{c}2014 \\
\text { Receiv- } \\
\text { able } \\
\text { Share }\end{array}$ & $\begin{array}{c}2016 \\
\text { Receiv- } \\
\text { able } \\
\text { turnov } \\
\text { er ratio }\end{array}$ & $\begin{array}{c}2015 \\
\text { Receiv- } \\
\text { able } \\
\text { turnov } \\
\text { er ratio }\end{array}$ & $\begin{array}{c}2014 \\
\text { Receiv- } \\
\text { able } \\
\text { turnov } \\
\text { er ratio }\end{array}$ \\
\hline 1 & 0,39 & 0,37 & 0,37 & 65 & 55 & 52 \\
\hline 2 & 0,28 & 0,34 & 0,47 & 47 & 65 & 71 \\
\hline 3 & 0,53 & 0,51 & 0,55 & 65 & 61 & 64 \\
\hline 4 & 0,74 & 0,79 & 0,75 & 80 & 90 & 97 \\
\hline 5 & 0,25 & 0,35 & 0,38 & 30 & 44 & 40 \\
\hline 6 & 0,50 & 0,51 & 0,53 & 90 & 83 & 99 \\
\hline 7 & 0,21 & 0,17 & 0,15 & 14 & 11 & 10 \\
\hline 8 & 0,36 & 0,36 & 0,30 & 50 & 48 & 41 \\
\hline 9 & 0,23 & 0,27 & 0,30 & 22 & 23 & 25 \\
\hline 10 & 0,18 & 0,18 & 0,29 & 15 & 18 & 47 \\
\hline 11 & 0,59 & 0,64 & 0,67 & 94 & 95 & 85 \\
\hline 12 & 0,39 & 0,40 & 0,49 & 39 & 36 & 45 \\
\hline 13 & 0,54 & 0,26 & 0,41 & 85 & 35 & 48 \\
\hline 14 & 0,33 & 0,58 & 0,64 & 55 & 100 & 122 \\
\hline 15 & 0,31 & 0,33 & 0,30 & 28 & 33 & 22 \\
\hline
\end{tabular}

\begin{tabular}{|c|c|c|c|c|c|c|}
\hline $\begin{array}{l}\text { Enterp } \\
\text { rise }\end{array}$ & $\begin{array}{c}2016 \\
\text { Receiv- } \\
\text { able } \\
\text { Share }\end{array}$ & $\begin{array}{c}2015 \\
\text { Receiv- } \\
\text { able } \\
\text { Share }\end{array}$ & $\begin{array}{c}2014 \\
\text { Receiv- } \\
\text { able } \\
\text { Share }\end{array}$ & $\begin{array}{c}2016 \\
\text { Receiv- } \\
\text { able } \\
\text { turnov } \\
\text { er ratio }\end{array}$ & $\begin{array}{c}2015 \\
\text { Receiv- } \\
\text { able } \\
\text { turnov } \\
\text { er ratio }\end{array}$ & $\begin{array}{c}2014 \\
\text { Receiv- } \\
\text { able } \\
\text { turnov } \\
\text { er ratio }\end{array}$ \\
\hline 16 & 0,74 & 0,76 & 0,74 & 93 & 116 & 120 \\
\hline 17 & 0,35 & 0,36 & 0,35 & 37 & 35 & 35 \\
\hline 18 & 0,57 & 0,54 & 0,59 & 73 & 67 & 68 \\
\hline 19 & 0,33 & 0,37 & 0,35 & 32 & 32 & 33 \\
\hline 20 & 0,57 & 0,49 & 0,55 & 82 & 35 & 49 \\
\hline 21 & 0,34 & 0,34 & 0,24 & 56 & 51 & 38 \\
\hline 22 & 0,35 & 0,39 & 0,43 & 51 & 66 & 64 \\
\hline 23 & 0,39 & 0,43 & 0,39 & 63 & 83 & 68 \\
\hline 24 & 0,49 & 0,50 & 0,40 & 91 & 88 & 70 \\
\hline 25 & 0,47 & 0,47 & 0,47 & 57 & 54 & 54 \\
\hline 26 & 0,42 & 0,51 & 0,43 & 66 & 118 & 85 \\
\hline 27 & 0,45 & 0,39 & 0,40 & 43 & 44 & 41 \\
\hline 28 & 0,21 & 0,24 & 0,39 & 54 & 65 & 80 \\
\hline 29 & 0,20 & 0,28 & 0,33 & 34 & 54 & 55 \\
\hline 30 & 0,64 & 0,65 & 0,58 & 94 & 144 & 105 \\
\hline 31 & 0,45 & 0,49 & 0,55 & 61 & 74 & 70 \\
\hline 32 & 0,32 & 0,29 & 0,30 & 40 & 35 & 40 \\
\hline 33 & 0,45 & 0,45 & 0,40 & 47 & 48 & 44 \\
\hline 34 & 0,32 & 0,34 & 0,37 & 48 & 41 & 47 \\
\hline 35 & 0,43 & 0,41 & 0,38 & 37 & 38 & 34 \\
\hline
\end{tabular}

c. Source: author's own study based on financial statements of enterprises

When analyzing both tables it can be seen that receivables constitute a high share in the structure of current assets. In the majority of enterprises operating in branch purchasing groups, receivables in the structure of current assets prevail. On the other hand, the receivables turnover rates in days are generally on an average level.Tables IV and $\mathrm{V}$ present the financial liquidity results in the analyzed enterprises in the years 20142016.

When analyzing Tables IV and V one can see that there are clear differences in the results of financial liquidity ratios in both groups. The significance of differences in the distribution of ratios between the branch and multi-branch group was assessed using the Mann-Whitney U test. Tables VI - IX present the characteristics of the distribution of individual ratios in both groups. Due to the fairly clear asymmetry in the distribution of all ratios (the table does not consider the skewness, but this is visible, for example, on the basis of a median and average comparison), the median will be a much more reliable measure of the average level. And it is the basis for deducing the differences between the two groups. As one can see, for most comparisons, the differences between the branch and multi-branch groups are statistically significant (the value of the test probability $p$ below 0.05 ). On the basis of the comparison of statistics in both groups, it can be stated that multi-branch companies are characterized by lower current financial liquidity and lower value of the fast liquidity ratio, as well as lower turnover. Only in the case of participation, there are no statistically significant differences between the two groups. The detailed results of the significance of differences in the distribution of ratios between the branch and multi-industry group using the Mann-Whitney U test are presented in Tables VI-IX. 
TABLE IV. RATIOS OF CURRENT FINANCIAL LIQUIDITY AND QUICK FINANCIAL LIQUIDITY IN THE SURVEYED ENTERPRISES OPERATING IN BRANCH PURCHASING GROUPS IN THE PERIOD 2014-2016

\begin{tabular}{|c|c|c|c|c|c|c|}
\hline $\begin{array}{l}\text { Enterpr } \\
\text { ise }\end{array}$ & $\begin{array}{c}2016 \\
\text { Current } \\
\text { liquidity } \\
\text { ratio }\end{array}$ & $\begin{array}{c}2015 \\
\text { Curren } \\
t \\
\text { liquidit } \\
\text { y ratio }\end{array}$ & $\begin{array}{c}2014 \\
\text { Curren } \\
t \\
\text { liquidit } \\
\text { y ratio }\end{array}$ & $\begin{array}{l}2016 \\
\text { Quick } \\
\text { ratio }\end{array}$ & $\begin{array}{c}2015 \\
\text { Quick } \\
\text { ratio }\end{array}$ & $\begin{array}{c}2014 \\
\text { Quic } \\
\mathbf{k} \\
\text { ratio }\end{array}$ \\
\hline 1 & 1.7 & 1.9 & 1.9 & 0.9 & 1 & 0.9 \\
\hline 2 & 11 & 9 & 7 & 6 & 4 & 4 \\
\hline 3 & 1.3 & 1.3 & 1.3 & 0.8 & 0.7 & 0.7 \\
\hline 4 & 3.7 & 3.4 & 3 & 2.1 & 2 & 1.9 \\
\hline 5 & 3.9 & 3.9 & 4.1 & 2 & 2.1 & 2.1 \\
\hline 6 & 1.5 & 1.4 & 1.5 & 1 & 1 & 1 \\
\hline 7 & 5.9 & 7 & 6.1 & 3.4 & 3.8 & 3.3 \\
\hline 8 & 1.8 & 1.9 & 1.8 & 1.1 & 1.2 & 1 \\
\hline 9 & 1.8 & 1.8 & 2 & 0.7 & 0.9 & 1 \\
\hline 10 & 3.6 & 4.7 & 4.6 & 1.7 & 1.5 & 1.3 \\
\hline 11 & 10 & 15 & 12 & 4.9 & 8.2 & 5.8 \\
\hline 12 & 2 & 1.8 & 1.7 & 0.2 & 0.3 & 0.2 \\
\hline 13 & 7.9 & 5.6 & 7.1 & 3.2 & 2.9 & 3.3 \\
\hline 14 & 2.1 & 1.9 & 1.5 & 1 & 0.9 & 0.8 \\
\hline 15 & 7.3 & 8.7 & 10.6 & 4.9 & 5.6 & 6.8 \\
\hline 16 & 3.4 & 3.6 & 3.5 & 2 & 2.1 & 2.1 \\
\hline 17 & 1.4 & 1.3 & 1.1 & 0.7 & 0.8 & 0.7 \\
\hline 18 & 1.6 & 1.6 & 1.4 & 0.9 & 0.9 & 0.8 \\
\hline 19 & 3.3 & 2.5 & 2.7 & 1.9 & 1.4 & 1.5 \\
\hline 20 & 7.7 & 7.8 & 7.5 & 3,4 & 3.2 & 2.9 \\
\hline 21 & 1.3 & 1.3 & 1.5 & 0.8 & 0.6 & 0.9 \\
\hline 22 & 1.3 & 1.5 & 1.6 & 0.6 & 0.6 & 0.7 \\
\hline 23 & 1.2 & 1.2 & 1.3 & 0.6 & 0.7 & 0.7 \\
\hline 24 & 1.4 & 1.5 & 1.5 & 0.7 & 0.8 & 0.8 \\
\hline 25 & 1.7 & 1.5 & 1.5 & 1 & 1 & 1 \\
\hline 26 & 1.7 & 2 & 1.8 & 1 & 1.2 & 1 \\
\hline 27 & 6.5 & 5.8 & 9.6 & 4.3 & 3.6 & 6.5 \\
\hline 28 & 1.4 & 1.2 & 1.3 & 0.9 & 0.8 & 0.8 \\
\hline 29 & 1.7 & 1.9 & 1,9 & 0.9 & 1 & 0.9 \\
\hline 30 & 11 & 9 & 7 & 6 & 4 & 4 \\
\hline 31 & 1.3 & 1,3 & 1.3 & 0.8 & 0.7 & 0.7 \\
\hline 32 & 3.7 & 3.4 & 3 & 2.1 & 2 & 1.9 \\
\hline 33 & 3.9 & 3.9 & 4.1 & 2 & 2.1 & 2.1 \\
\hline 34 & 1.5 & 1.4 & 1.5 & 1 & 1 & 1 \\
\hline 35 & 5.9 & 7 & 6.1 & 3.4 & 3.8 & 3.3 \\
\hline
\end{tabular}

TABLE V. RATIOS OF CURRENT FINANCIAL LIQUIDITY AND QUICK FINANCIAL LIQUIDITY IN THE SURVEYED ENTERPRISES OPERATING IN MULTIBRANCH PURCHASING GROUPS IN THE PERIOD 2014-2016

\begin{tabular}{|l|l|l|l|l|l|l|}
\hline $\begin{array}{c}\text { Enterp } \\
\text { rise }\end{array}$ & $\begin{array}{c}\mathbf{2 0 1 6} \\
\text { Curren } \\
\mathbf{t} \\
\text { lquidit } \\
\text { y ratio }\end{array}$ & $\begin{array}{c}\mathbf{2 0 1 5} \\
\text { Curren } \\
\mathbf{t} \\
\text { liquidit } \\
\text { y ratio }\end{array}$ & $\begin{array}{c}\mathbf{2 0 1 4} \\
\text { Curren } \\
\mathbf{t} \\
\text { liquidit } \\
\text { y ratio }\end{array}$ & $\begin{array}{c}\mathbf{2 0 1 6} \\
\text { Quick } \\
\text { ratio }\end{array}$ & $\begin{array}{c}\mathbf{2 0 1 5} \\
\text { Quick } \\
\text { ratio }\end{array}$ & $\begin{array}{c}\mathbf{2 0 1 4} \\
\text { Quick } \\
\text { ratio }\end{array}$ \\
\hline 1 & 2,3 & 2 & 2,2 & 1 & 0,7 & 0,8 \\
\hline 2 & 4 & 3,6 & 3,1 & 1,1 & 1,2 & 1,4 \\
\hline 3 & 2 & 1,9 & 1,8 & 1,1 & 1 & 1 \\
\hline 4 & 1,1 & 1,1 & 1,2 & 0,8 & 0,9 & 0,8 \\
\hline 5 & 1,8 & 1,6 & 1,6 & 0,6 & 0,6 & 0,6 \\
\hline 6 & 1,6 & 1,7 & 1,6 & 0,8 & 0,8 & 0,8 \\
\hline 7 & 1,6 & 1,6 & 1,5 & 0,5 & 0,4 & 0,4 \\
\hline 8 & 2 & 2,3 & 2,7 & 0,8 & 1 & 1,3 \\
\hline 9 & 2,1 & 2,3 & 1,9 & 0,9 & 0,9 & 0,9 \\
\hline 10 & 2,9 & 2,4 & 2,2 & 0,7 & 0,7 & 0,9 \\
\hline 11 & 1,1 & 1 & 1 & 0,7 & 0,8 & 0,8 \\
\hline 12 & 1,4 & 1,4 & 1,4 & 0,6 & 0,6 & 0,7 \\
\hline 13 & 1,4 & 1,8 & 2 & 0,7 & 0,8 & 0,5 \\
\hline 14 & 1,8 & 2 & 1,3 & 0,8 & 1,3 & 0,9 \\
\hline 15 & 1,1 & 0,8 & 0,8 & 0,3 & 0,3 & 0,3 \\
\hline
\end{tabular}

\begin{tabular}{|l|l|l|l|l|l|l|}
\hline 16 & 1,3 & 1,4 & 1,3 & 1 & 1 & 1 \\
\hline 17 & 12 & 12 & 9 & 6 & 6 & 5 \\
\hline 18 & 1,3 & 1,3 & 1,4 & 0,7 & 0,7 & 0,8 \\
\hline 19 & 1 & 1 & 1 & 0,3 & 0,3 & 0,4 \\
\hline 20 & 1,4 & 1,2 & 1 & 0,8 & 0,7 & 0,6 \\
\hline 21 & 2,3 & 2,8 & 2,3 & 1,2 & 1,4 & 0,7 \\
\hline 22 & 4 & 2,6 & 4,1 & 1,6 & 1,2 & 2 \\
\hline 23 & 3,3 & 2,3 & 3 & 1,5 & 1,3 & 1,4 \\
\hline 24 & 1,2 & 1,2 & 1,3 & 0,6 & 0,6 & 0,5 \\
\hline 25 & 1,4 & 1,4 & 1,3 & 0,7 & 0,6 & 0,7 \\
\hline 26 & 1,3 & 1,2 & 1,7 & 0,6 & 0,7 & 0,9 \\
\hline 27 & 1,8 & 1,5 & 1,8 & 0,8 & 0,6 & 0,7 \\
\hline 28 & 7,1 & 6 & 5 & 5,8 & 4,5 & 4,1 \\
\hline 29 & 0,6 & 0,8 & 1,1 & 0,1 & 0,3 & 0,3 \\
\hline 30 & 3 & 1,9 & 2,5 & 2,2 & 1,4 & 1,7 \\
\hline 31 & 1,7 & 1,6 & 1,7 & 0,8 & 0,8 & 0,9 \\
\hline 32 & 2,5 & 3,1 & 2,7 & 1 & 1,1 & 1,3 \\
\hline 33 & 1,4 & 1,3 & 1,2 & 0,8 & 0,7 & 0,5 \\
\hline 34 & 1,6 & 1,5 & 1,6 & 0,6 & 0,6 & 0,5 \\
\hline 35 & 0,8 & 0,8 & 0,9 & 0,37 & 0,33 & 0,35 \\
\hline
\end{tabular}

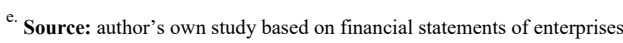

TABLE VI. THE RESULTS OF THE RATIOS OF THE SHARE OF RECEIVABLES IN CURRENT ASSETS IN THE BRANCH AND MULTI-BRANCH GROUP

\begin{tabular}{|c|c|c|c|c|c|c|c|c|c|c|c|}
\hline \multirow{3}{*}{ Year } & \multicolumn{10}{|c|}{ Group } & \multirow{3}{*}{$p$} \\
\hline & \multicolumn{5}{|c|}{ Branch $(N=35)$} & \multicolumn{5}{|c|}{ Multi-branch $(N=35)$} & \\
\hline & $\bar{x}$ & $\mathrm{Me}$ & $S$ & $\min$ & $\begin{array}{l}\mathrm{ma} \\
\mathrm{x}\end{array}$ & $\bar{x}$ & $\mathrm{Me}$ & $s$ & $\min$ & \begin{tabular}{|l}
$\mathrm{ma}$ \\
$\mathrm{x}$
\end{tabular} & \\
\hline 2014 & $\begin{array}{l}0,4 \\
7\end{array}$ & $\begin{array}{l}0,5 \\
0\end{array}$ & $\begin{array}{l}0,1 \\
3\end{array}$ & \begin{tabular}{|l|}
0,1 \\
4
\end{tabular} & $\begin{array}{l}0,7 \\
4\end{array}$ & $\begin{array}{l}0,4 \\
4\end{array}$ & $\begin{array}{l}0,4 \\
0\end{array}$ & $\begin{array}{l}0,1 \\
4\end{array}$ & \begin{tabular}{|l|}
0,1 \\
5
\end{tabular} & $\begin{array}{l}0,7 \\
5\end{array}$ & $\begin{array}{l}0,0 \\
979\end{array}$ \\
\hline 2015 & $\begin{array}{l}0,4 \\
6\end{array}$ & $\begin{array}{l}0,4 \\
8\end{array}$ & $\begin{array}{l}0,1 \\
4\end{array}$ & $\begin{array}{l}0,1 \\
3\end{array}$ & $\begin{array}{l}0,7 \\
1\end{array}$ & $\begin{array}{l}0,4 \\
2\end{array}$ & $\begin{array}{l}0,3 \\
9\end{array}$ & $\begin{array}{l}0,1 \\
5\end{array}$ & $\begin{array}{l}0,1 \\
7\end{array}$ & $\begin{array}{l}0,7 \\
9\end{array}$ & $\begin{array}{l}0,1 \\
215\end{array}$ \\
\hline 2016 & $\begin{array}{l}0,4 \\
5 \\
\end{array}$ & $\begin{array}{l}0,4 \\
7 \\
\end{array}$ & $\begin{array}{l}0,1 \\
4\end{array}$ & $\begin{array}{l}0,0 \\
9\end{array}$ & $\begin{array}{l}0,7 \\
0\end{array}$ & $\begin{array}{l}0,4 \\
1\end{array}$ & $\begin{array}{l}0,3 \\
9\end{array}$ & $\begin{array}{l}0,1 \\
5\end{array}$ & \begin{tabular}{|l|}
0,1 \\
8
\end{tabular} & \begin{tabular}{|l|l}
0,7 \\
4
\end{tabular} & $\begin{array}{l}0,1 \\
079\end{array}$ \\
\hline
\end{tabular}

TABLE VII. THE OF CURRENT FINANCIAL LIQUIDITY RATIOS IN THE BRANCH AND MULTI-BRANCH GROUP

\begin{tabular}{|c|c|c|c|c|c|c|c|c|c|c|c|}
\hline \multirow{3}{*}{$\begin{array}{c}\text { Yea } \\
\mathbf{r}\end{array}$} & \multicolumn{10}{|c|}{ Group } & \multirow{3}{*}{$p$} \\
\hline & \multicolumn{5}{|c|}{ Branch $(N=35)$} & \multicolumn{5}{|c|}{ Multi-branch $(N=35)$} & \\
\hline & $\bar{x}$ & $\mathrm{Me}$ & $s$ & $\begin{array}{l}\mathrm{mi} \\
\mathrm{n}\end{array}$ & \begin{tabular}{|l|}
$\mathrm{ma}$ \\
$\mathrm{x}$
\end{tabular} & $\bar{x}$ & $\mathrm{Me}$ & $s$ & $\begin{array}{l}\mathrm{mi} \\
\mathrm{n}\end{array}$ & $\begin{array}{l}\mathrm{ma} \\
\mathrm{x}\end{array}$ & \\
\hline $\begin{array}{l}201 \\
4\end{array}$ & $\begin{array}{l}3,4 \\
9\end{array}$ & 2,2 & \begin{tabular}{|l|}
2,8 \\
7
\end{tabular} & 1,1 & $\begin{array}{l}12, \\
0\end{array}$ & \begin{tabular}{|l|}
2,0 \\
6
\end{tabular} & 1,6 & \begin{tabular}{|l|}
1,5 \\
1
\end{tabular} & 0,8 & 9,0 & $\begin{array}{l}0,009 \\
2 * *\end{array}$ \\
\hline $\begin{array}{l}201 \\
5 \\
\end{array}$ & $\begin{array}{l}3,5 \\
2 \\
\end{array}$ & 2,2 & \begin{tabular}{|l|}
2,9 \\
8 \\
\end{tabular} & 1,2 & $\begin{array}{l}15, \\
0 \\
\end{array}$ & \begin{tabular}{|l|}
2,1 \\
3 \\
\end{tabular} & 1,6 & \begin{tabular}{|l|}
1,9 \\
8 \\
\end{tabular} & 0,8 & $\begin{array}{l}12, \\
0\end{array}$ & \begin{tabular}{|l|}
0,006 \\
$9 * *$ \\
\end{tabular} \\
\hline $\begin{array}{l}201 \\
6\end{array}$ & $\begin{array}{l}3,3 \\
5\end{array}$ & 2,0 & $\begin{array}{l}2,6 \\
4\end{array}$ & 1,2 & \begin{tabular}{|l}
11, \\
0
\end{tabular} & $\begin{array}{l}2,2 \\
6\end{array}$ & 1,6 & \begin{tabular}{|l|}
2,0 \\
8
\end{tabular} & 0,6 & $\begin{array}{l}12, \\
0\end{array}$ & \begin{tabular}{|l|}
0,023 \\
$5^{*}$
\end{tabular} \\
\hline
\end{tabular}

TABLE VIII. THE RESULTS OF QUICK FINANCIAL LIQUIDITY RATIOS IN THE BRANCH AND MULTI-BRANCH GROUP

\begin{tabular}{|c|c|c|c|c|c|c|c|c|c|c|c|}
\hline \multirow{3}{*}{$\begin{array}{c}\text { Yea } \\
\mathbf{r}\end{array}$} & \multicolumn{10}{|c|}{ Group } & \multirow{3}{*}{$p$} \\
\hline & \multicolumn{5}{|c|}{ Branch $(N=35)$} & \multicolumn{5}{|c|}{ Multi-branch $(N=35)$} & \\
\hline & $\bar{x}$ & $\mathrm{Me}$ & $s$ & $\min$ & $\begin{array}{l}\mathrm{ma} \\
\mathrm{x}\end{array}$ & $\bar{x}$ & $\mathrm{Me}$ & $s$ & $\begin{array}{l}\mathrm{mi} \\
\mathrm{n}\end{array}$ & $\begin{array}{l}\mathrm{ma} \\
\mathrm{x}\end{array}$ & \\
\hline $\begin{array}{l}201 \\
4 \\
\end{array}$ & $\begin{array}{l}1,7 \\
8 \\
\end{array}$ & 1,0 & \begin{tabular}{|l|}
1,6 \\
8 \\
\end{tabular} & 0,2 & 6,8 & $\begin{array}{l}1,0 \\
4 \\
\end{array}$ & 0,8 & $\begin{array}{l}0,9 \\
6 \\
\end{array}$ & \begin{tabular}{|l|}
0,3 \\
0 \\
\end{tabular} & 5,0 & $\begin{array}{l}0,005 \\
7 * *\end{array}$ \\
\hline $\begin{array}{l}201 \\
5 \\
\end{array}$ & $\begin{array}{l}1,8 \\
0\end{array}$ & 1,2 & \begin{tabular}{|l|}
1,6 \\
3 \\
\end{tabular} & 0,3 & 8,2 & $\begin{array}{l}1,0 \\
5\end{array}$ & 0,8 & $\begin{array}{l}1,1 \\
1\end{array}$ & \begin{tabular}{|l|}
0,3 \\
0 \\
\end{tabular} & 6,0 & $\begin{array}{l}0,001 \\
5 * *\end{array}$ \\
\hline $\begin{array}{l}201 \\
6\end{array}$ & $\begin{array}{l}1,7 \\
3\end{array}$ & 1,0 & \begin{tabular}{|l|}
1,4 \\
5
\end{tabular} & 0,2 & 6,0 & $\begin{array}{l}1,1 \\
1\end{array}$ & 0,8 & $\begin{array}{l}1,2 \\
6\end{array}$ & \begin{tabular}{|l|}
0,1 \\
0
\end{tabular} & 6,0 & $\begin{array}{l}0,005 \\
7 * *\end{array}$ \\
\hline
\end{tabular}


TABLE IX. THE RESULTS OF RECEIVABLES TURNOVER RATES IN DAYS IN THE BRANCH AND MULTI-BRANCH GROUP

\begin{tabular}{|c|c|c|c|c|c|c|c|c|c|c|c|}
\hline \multirow{3}{*}{$\begin{array}{c}\text { Yea } \\
\mathbf{r}\end{array}$} & \multicolumn{10}{|c|}{ Group } & \multirow{3}{*}{$p$} \\
\hline & \multicolumn{5}{|c|}{ Branch $(N=35)$} & \multicolumn{5}{|c|}{ Multi-branch $(N=35)$} & \\
\hline & $\bar{x}$ & $\mathrm{Me}$ & $S$ & $\begin{array}{l}\mathrm{mi} \\
\mathrm{n}\end{array}$ & $\begin{array}{l}\mathrm{ma} \\
\mathrm{x}\end{array}$ & $\bar{x}$ & $\mathrm{Me}$ & $S$ & $\begin{array}{l}\mathrm{mi} \\
\mathrm{n}\end{array}$ & $\begin{array}{l}\mathrm{ma} \\
\mathrm{x}\end{array}$ & \\
\hline $\begin{array}{l}201 \\
4\end{array}$ & $\begin{array}{l}68, \\
0\end{array}$ & $\begin{array}{l}69, \\
0\end{array}$ & $\begin{array}{l}21, \\
4\end{array}$ & 18 & 122 & $\begin{array}{l}59, \\
1\end{array}$ & $\begin{array}{l}52, \\
0\end{array}$ & $\begin{array}{l}27, \\
0\end{array}$ & 10 & $\begin{array}{l}12 \\
2\end{array}$ & $\begin{array}{l}0,05 \\
83\end{array}$ \\
\hline $\begin{array}{l}201 \\
5\end{array}$ & $\begin{array}{l}67, \\
4\end{array}$ & $\begin{array}{l}74, \\
0\end{array}$ & $\begin{array}{l}22, \\
3\end{array}$ & 14 & 113 & $\begin{array}{l}59, \\
6\end{array}$ & $\begin{array}{l}\text { 54, } \\
0\end{array}$ & $\begin{array}{l}30, \\
4\end{array}$ & 11 & $\begin{array}{l}14 \\
4\end{array}$ & $\begin{array}{l}0,09 \\
55\end{array}$ \\
\hline $\begin{array}{l}201 \\
6\end{array}$ & $\begin{array}{l}70, \\
5\end{array}$ & $\begin{array}{l}72, \\
0\end{array}$ & $\begin{array}{l}23, \\
3\end{array}$ & 11 & 132 & $\begin{array}{l}55, \\
4\end{array}$ & $\begin{array}{l}54, \\
0\end{array}$ & $\begin{array}{l}23, \\
1\end{array}$ & 14 & 94 & $\begin{array}{l}0,00 \\
85^{*} \\
*\end{array}$ \\
\hline
\end{tabular}

i. $p$-test probability value calculated by means of a test

Then, the correlations between the various indicators were also examined using for this purpose the Spearman's rank correlation coefficient. The calculations were made without any division into a group of branch and multi-branch enterprises. The details are presented in Table X.

TABLE X. .SPEARMAN'S RANK CORRELATION COEFFICIENT FOR THE ANALYZED RATIOS IN 2016

\begin{tabular}{|c|c|c|c|c|}
\hline 2016 & $\begin{array}{c}\text { Receivable } \\
\text { s } \\
\text { share }\end{array}$ & $\begin{array}{l}\text { Financial } \\
\text { liquidity }\end{array}$ & $\begin{array}{l}\text { Qucik } \\
\text { ratio }\end{array}$ & $\begin{array}{c}\text { Receivable } \\
\text { turnover }\end{array}$ \\
\hline $\begin{array}{l}\text { Receivables } \\
\text { share }\end{array}$ & 1 & $\begin{array}{l}-0,30 \\
\left(0,0118^{*}\right)\end{array}$ & $\begin{array}{l}0,12 \\
(0,3164)\end{array}$ & $\begin{array}{l}0,84 \\
(0,0000 * * *)\end{array}$ \\
\hline $\begin{array}{c}\text { Financial } \\
\text { liquidity }\end{array}$ & $\begin{array}{l}-0,30 \\
(0,0118 *)\end{array}$ & 1 & $\begin{array}{l}0,85 \\
(0,0000 * * *)\end{array}$ & $\begin{array}{l}-0,04 \\
(0,7317)\end{array}$ \\
\hline $\begin{array}{l}\text { Quick } \\
\text { ratio }\end{array}$ & $\begin{array}{l}0,12 \\
(0,3164)\end{array}$ & $\begin{array}{l}0,85 \\
(0,0000 * * *)\end{array}$ & 1 & $\begin{array}{l}0,34 \\
(0,0037 * *)\end{array}$ \\
\hline $\begin{array}{l}\text { Receivable } \\
\text { turnover }\end{array}$ & $\begin{array}{l}0,84 \\
(0,0000 * * *) \\
\end{array}$ & $\begin{array}{l}-0,04 \\
(0,7317) \\
\end{array}$ & $\begin{array}{l}0,34 \\
(0,0037 * *)\end{array}$ & 1 \\
\hline
\end{tabular}

The high correlation in 2016 showed a turnover and a share $(\mathrm{R}=0.84)$ as well as Quick ratio and liquidity $(\mathrm{R}=0.85)$. These correlations are quite high; one can say that in the description of the financial condition of companies, the ratio is somewhat interchangeable. In 2015 and 2014 correlation rates were at a similar level for the same ratios as in 2016

\section{CONCLUSION}

The use of the above methods and tools supporting the process of managing receivables is more possible in enterprises operating in branch group purchasing organizations. Especially using the economies of scale allows getting a favorable trade credit. It later becomes an important element of the receivables management policy. Enterprises do not have to pay their liabilities quickly so they can extend the repayment date for their recipients. The materiality test showed that the difference in the receivables turnover in days was statistically significant. Enterprises operating in the branch purchasing groups charge more slowly in comparison to companies operating in multibranch groups. It is a conscious policy of managing receivables, but not an inept debt collection policy. In the purchasing groups it is also easier to apply benchmarking. It is also more effective to monitor recipients collectively if enterprises operate in the same branch. This allows reducing the risk of occurrence of overdue receivables. The same applies to mutual transactions which are designed to improve financial safety. When analyzing the ratio of current and quick financial liquidity, there are high differences which are statistically significant. An excess in liquidity appears in companies operating in branch purchasing groups. These enterprises also have high quick liquidity, which indicates a high level of receivables in the structure of current assets. Indeed, the share of receivables in current assets in the entities forming branch purchasing groups is high. In 24 enterprises operating in branch groups they constitute the largest share.

To sum up, companies operating in branch purchasing groups have excess liquidity but do not use aggressive policy of debt collection as evidenced by higher receivables turnover rates in days. The share of receivables over inventories is also higher. These results indicate moderate - conservative strategies for managing receivables. On the other hand, enterprises operating in multi-branch purchasing groups are also characterized by high liquidity, but definitely lower in the previous group. In the case of 25 enterprises, the share of inventories in current assets is higher than receivables. In addition, debts are collected quickly. All these elements indicate the conservative strategy of receivables management.

However, in both purchasing groups managers should continue an implementation of additional solutions that will lead to an improvement in receivables turnover in days.

\section{REFERENCES}

[1] M. Sierpińska and D.Wędzki, Zarządzanie płynnością finansową w przedsiębiorstwie, Warszawa: PWN, 2010, pp. 230,

[2] G. Zimon, An analysis of level of net working capital in companies operating in purchasing groups. Conference: 20th International Scientific Conference on Economic and Social Development (ESD) Location: Prague, Czech Republic Date: APR 27-28,2017 Economic and Social Development (ESD) Book Series: International Scientific Conference on Economic and Social Development, 2017, pp. 531-537.

[3] E.I. Altman and E. Hotchkiss, Trudności finansowe a upadłość firm. Warszawa: CedeWu, 2006, pp. 236.

[4] W. Bień, Zarządzanie finansami przedsiębiorstwa, Warszawa: Difin, 2008, pp. 240.

[5] G. Zimon, "Influence of group purchasing organizations on financial situation of Polish SMEs," Oeconomia Copernicana, vol. 9(1), pp.87104 , March 2018.

[6] R.D Blair and C.P Durrance, "Group purchasing organizations, monopsony, and antitrust Policy," Managerial and Decision Economics, vol. 35(7), pp. 23-30, 2014.

[7] M. Porter, Porter o konkurencji, Warszawa, PWE, 2001, pp.37.

[8] E. Tella and V.M Virolainen, "Motives behind purchasing consortia," International Journal of Production Economics, pp.161-168, 2005.

[9] J. Nollet and M. Beaulieu, Should an organization join a purchasing group?" Supply Chain Management, vol. 10(1), pp.1-17, 2005.

[10] L.R Burns and J.A Lee, "Hospital purchasing alliances: Utilization, services, and performance," Health Care Management Review: July/September, vol. 33(3), pp.7-17,2008.

[11] A.E. Cowan, S.J. Clark, J.L. Gordon, K. Bok, and A. K. Shen, Vaccine purchasing groups in the United States: An overview of their policies and practices, vol. 34 (2016), pp. 5060-5065.

[12] G. Zimon, "Organization of Transport in a Commercial Enterprise Operating in a Branch Purchasing Group," Estudios de Economia Aplicada, vol. 36-3, pp. 811-824, 2018. 